\title{
Semantic fluency reveals reduced functional connectivity between subcategorical co-hyponyms in recent-onset inpatients with first-episode psychosis
}

\section{Petar Gabrić \& Mija Vandek}

To cite this article: Petar Gabrić \& Mija Vandek (2021): Semantic fluency reveals reduced functional connectivity between subcategorical co-hyponyms in recent-onset inpatients with firstepisode psychosis, Clinical Linguistics \& Phonetics, DOI: 10.1080/02699206.2021.1961019

To link to this article: https://doi.org/10.1080/02699206.2021.1961019

曲 Published online: 06 Aug 2021.

Submit your article to this journal $₫$

Q View related articles $\sqsubset$

View Crossmark data \lceil 
This is an original manuscript of an article published by Taylor \& Francis in Clinical $\begin{array}{llllll}\text { Linguistics \& Phonetics on } 06 \text { Aug 2021, available online: } & \text { \& }\end{array}$ https://www.tandfonline.com/doi/full/10.1080/02699206.2021.1961019

If you do not have access to the published version, please contact Petar Gabrić: petar.gabric@uni-marburg.de or petar.gabric1@gmail.com

Semantic fluency reveals reduced functional connectivity between subcategorical cohyponyms in recent-onset inpatients with first-episode psychosis

Petar GABRIĆa, ${ }^{\text {, }}$ Mija VANDEK ${ }^{\mathrm{a}}$

${ }^{a}$ Department of Linguistics, University of Zagreb, Croatia

${ }^{\mathrm{b}}$ Institute for German Linguistics, Philipps University of Marburg, Germany

Corresponding author: Petar Gabrić, Institute for German Linguistics, Philipps University of Marburg, Pilgrimstein 16, 35032 Marburg an der Lahn, Germany; email: petar.gabric@unimarburg.de, petar.gabric1@gmail.com

Petar Gabrić ORCiD 0000-0003-2644-194X

Mija Vandek ORCiD 0000-0003-3398-7350 


\begin{abstract}
Data on clustering and switching during semantic fluency (SF) in patients with first-episode psychosis (PwFEP) are scant. We aimed to investigate (1) clustering and switching on SF in PwFEP using more detailed clustering analyses and (2) the possibility of disproportionate clustering deficits across different SF tasks in PwFEP and healthy subjects (HS), with the latter being suggested by the current literature on patients with schizophrenia. We recruited 22 Croatianspeaking PwFEP with schizophrenia features or symptoms and 22 HS matched in age, sex distribution, and handedness. All patients were medicated and had a mean illness duration of 1 month. The categories animals, trees, vegetables, fruits, and musical instruments were administered for SF. PwFEP produced significantly fewer correct words in the aggregate score, as well as across all categories. The switching rate was significantly higher in PwFEP, but no post hoc comparisons were significant. PwFEP also produced significantly smaller clusters, yet the post hoc comparisons for the tree and fruit task were not significant. A higher switching rate and smaller clusters indicate less efficient functional connectivity within subcategories of the given categories, but not necessarily between the subcategories. Although both less likely to produce a cluster once a switch has been uttered and less likely to produce clusters larger than two words compared to HS, the latter deficit was more pronounced. Our results further suggest that PwFEP might show normal clustering performance on some SF tasks. We discuss the results in the context of the hypothesis of semantic hyperactivation in psychoses.
\end{abstract}

Keywords: semantic fluency; schizophrenia; first-episode psychosis; clustering; hypernymy; semantic memory 


\section{Background}

\subsection{Clustering and switching on semantic fluency}

Verbal fluency tasks are commonly utilized in both clinical practice and research to assess the level of cognitive, linguistic, or communicative impairment in patients with brain damage or psychiatric disorders (Pekkala 2012; Thiele et al. 2016). In a semantic fluency (SF) task, the subject is to name as many members of a given semantic category (e.g. animals) within a time limit. Despite its frequent use, the cognitive and specific linguistic correlates of SF remain mostly unclear. Although the presumed working memory and executive functioning components of both SF and letter fluency are often emphasized (Amunts et al., 2020), studies suggest that both general cognitive and specific linguistic processing subserve performance on verbal fluency (Fong et al., 2020; Stielow \& Stenneken, 2017). Nonetheless, which linguistic (or specifically semantic) processes subserve verbal fluency remains likewise unclear. Functional imaging, cortical modulation, and lesion studies suggest that performance on SF is mediated by the metabolic activity and structural integrity of the bilateral inferior frontal gyri and left temporal cortex (Biesbroek et al., 2016; Nagels et al., 2012).

Performance on SF is often interpreted using only the raw number of correct words. However, it has been observed that subjects engaged in a verbal fluency task employ two specific cognitive processes: clustering and switching (Troyer et al., 1997). Clustering relates to the tendency of subjects to successively produce words denoting concepts belonging to the same subcategory (i.e. subcategorical co-hyponyms; e.g. reptiles) of the given semantic category (e.g. animals). 
Switching stands for the change of the semantic subcategory of the given category. For example, if a subject starts the animal task with the output dog-cat-kangaroo-whale-shark, we will count three accessed categories (pets, kangaroo, and marine animals), two clusters (pets and marine animals), two switches (pets-kangaroo and kangaroo-marine animals), and four clustered words (dog, cat, whale, and shark). Described as such, clustering and switching reflect the functional connectivity both within semantic subcategories (clustering) and between semantic subcategories (switching) of the given category in the semantic memory. Clustering and switching have been described as relatively automatic and controlled processes, respectively (Troyer \& Moscovitch, 2006). Recent research shows that clustering and switching on SF are correlated with matrix reasoning (reflects the ability to apply knowledge in resolving a novel problem; Raven's Standard Progressive Matrices) and processing speed (Fong et al., 2020). Nonetheless, Vandek et al. (2018) and Gabrić and Vandek (2020) found that clustering and switching on the tree, but not the animal task were associated with cognitive flexibility, indicating the existence of category specificities in clustering and switching.

\subsection{Prior studies in patients with schizophrenia}

Patients with schizophrenia regularly perform significantly poorer on SF compared to healthy subjects (Chen et al., 2020; Gourovitch et al., 1996; Joyce et al., 1996; Lemieux \& Macoir, 2017; Neill et al., 2014; Phillips et al., 2004; Rosenkranz et al., 2019; Rossell et al., 1999; Tyburski et al., 2015; van Beilen et al., 2004). Because studies suggest that patients with schizophrenia exhibit abnormally broad semantic spreading activation on semantic tasks compared to healthy subjects (Kuperberg et al., 2018; Spitzer, 1997), a plausible hypothesis would be that patients with 
schizophrenia will both switch at higher rates and produce smaller clusters on SF compared to healthy subjects, reflecting their presumed diminished capacity for localized semantic spreading activation (or greater capacity for broader semantic spreading activation). However, results regarding clustering and switching have been mixed and difficult to interpret, presumably due to methodological differences across studies and deficiencies in the quality of the statistical procedures in some studies. Several studies compared the raw values of clusters, clustered words (CW), and switches (SW) without controlling for total output, thus failing to capture the specific contributions of clustering and switching in the SF deficits in their samples (Batty et al., 2015; Elvevåg et al., 2002; Kosmidis et al., 2005; Moore et al., 2006; Rinaldi et al., 2013; Robert et al., 1998; Veleva et al., 2019). Studies that analyzed either CW or SW while controlling for total output yielded no significant differences between groups (SW: Berberian et al., 2016; Landrø \& Ueland, 2008, Okruszek et al., 2013; both CW and SW: Bozikas et al., 2005), significantly fewer clustered words in the patient group (Allen et al., 1993; Berberian et al., 2016), or significantly higher switching in patients with hallucinations but not without hallucinations (Popescu \& Micluţia, 2006). Additionally, a recent meta-analysis found that patients produce smaller clusters on SF (Tan et al., 2020, 2021) but the methods used in the study have been criticized (Gabrić 2021a, 2021b). Thus, (1) there is no evidence that patients with schizophrenia switch either significantly more or significantly less than healthy subjects (when controlling for the total output), and (2) the results regarding cluster magnitude are mixed. Notably, regarding clustering on 60-second SF tasks, Berberian et al. (2016) used only the animal task, while Bozikas et al. (2005) analyzed the aggregate score for the categories animals, fruits, and objects, pointing to possible categoryspecific effects on cluster magnitude on SF in patients with schizophrenia. Importantly, previous research on neurological disorders (such as Alzheimer's and Parkinson's diseases) has 
demonstrated category-specific deficits in clustering on SF (Moreno-Martínez et al., 2017; Neves et al., 2020; Tagini et al., 2018), indicating the possibility of the existence of similar phenomena in psychiatric disorders.

Another approach has been to calculate the so-called 'cluster size'. Most studies have employed the calculation for cluster size as proposed by Troyer et al. (1997): $\left[=\right.$ ( $_{\text {clustered words- }}{ }^{-}$ $\left.\left.\mathrm{n}_{\text {clusters }}\right) / \mathrm{n}_{\text {switches }}\right]$. However, this method yields a variable that simultaneously gives information on (1) the relative frequency of clustering events compared to switching events (i.e. the likelihood of building a cluster once a switch has been produced) and (2) the number of words listed within a cluster once a cluster has been produced (e.g. the likelihood that a cluster will continue after two words have been clustered before switching again). Studies that calculated cluster size as proposed by Troyer et al. (1997) found no significant differences between patients with schizophrenia and healthy subjects (Elvevåg et al., 2002; Landrø \& Ueland, 2008; Moore et al., 2006; Okruszek et al., 2013). Additionally, studies using semantic space modelling of the SF output report that the output of patients with schizophrenia is semantically disorganized compared to healthy controls, indicating dysfunctional connectivity between concepts in the semantic memory (Aloia et al., 1996; Berto \& Galaverna, 2016; Paulsen et al., 1996; Popescu \& Micluţia, 2006; Popescu et al. 2007; Sumiyoshi et al., 2009; Sung et al., 2012).

\subsection{Prior studies in patients with first-episode psychosis}

Patients with first-episode psychosis include patients in the early stages of psychosis, i.e. patients who have relatively recently started experiencing psychotic symptoms and otherwise have no known history of psychosis (Breitborde et al., 2009). Patients with first-episode psychosis may 
show similar levels of psychotic symptoms and cognitive deficits as patients with chronic schizophrenia (Reed, 2008). If the symptoms persist for longer periods, the patients may be diagnosed with schizophrenia (Menezes et al., 2006; cf. WHO, 1993: 79). Similarly to chronic patients, patients with first-episode psychosis (PwFEP) produce significantly fewer correct words than healthy subjects on SF tasks (Blessing et al., 2009; Giovannetti et al., 2003; Nuyen et al., 2005). Giovannetti et al. (2003) is the only study we encountered that analyzed clustering and switching. They found no significant differences between groups in cluster size on animal fluency. However, contrary to Troyer et al. (1997), they employed an arbitrary formal semantic-featurebased system for clustering in which two successive words could be classified as clusters if they shared features (e.g. small, herbivore) in at least four out of six feature categories (e.g. size, diet). Problematically, (1) studies using computational approaches have not suggested that organization of the output on animal fluency can be classified according to all these features (Aloia et al., 1996; Paulsen et al., 1996; Popescu \& Micluţia, 2006), (2) there appears to be convergence across some feature categories (e.g. geographical location and habitat, zoological class and biological order, etc.), while (3) the higher number of categorization decisions needed to be made by the experimenter for every word (instead of one categorization decision for two successive words) arguably increases the risk of error.

The aims of the present study were (1) to investigate clustering and switching on SF in PwFEP, as previous research has been scant, (2) to investigate possible category-specific deficits in cluster magnitude on SF in PwFEP, as they are a possible reason for the currently disproportionate results across the few studies conducted, and (3) to separately examine the switching rate and cluster magnitude in PwFEP. To disentangle these two components of 'cluster size', we calculated (1) the 
switching rate $\left[=\left(\mathrm{n}_{\text {switches }}+1-\mathrm{n}_{\text {clusters }}\right) /\left(\mathrm{n}_{\text {switches }}+1\right)\right]^{1}$ (as also used in e.g. Popescu \& Micluţia 2006) and (2) cluster magnitude ( $=\mathrm{n}_{\text {clustered words }} / \mathrm{n}_{\text {clusters }}$ ) (as also used in e.g. Batty et al. 2015; Giovanetti et al. 2003). Calculated as such, the switching rate can range from 0 to 1 , with a value of 0 possible only if the subject produced a cluster with the first subcategory they accessed and did not switch to another subcategory (due to continuous production within the first subcategory, silence, or alternations between the two), while a value of 1 implies the subject built absolutely no clusters. Cluster magnitude can range from 1 (if the subject built no clusters) or 2 (the minimum size of a cluster, as per its definition), while the maximum cluster magnitude is unknown and restricted by human physiology, subject's vocabulary size, and the task time limit. Presumably, this approach allows us an inspection of the efficiency of the connectivity between the more abstract and schematized co-hyponymic subcategories of a given category (switching rate; e.g. co-hyponyms birds-reptiles-farm animals of the category animals), as well as within the subcategories where more concrete concepts are situated (cluster magnitude; e.g. co-hyponyms elephant-lioncrocodile of the subcategory African animals) in the semantic memory of PwFEP.

\section{Materials and methods}

\subsection{Subjects}

22 Croatian-speaking inpatients diagnosed with an acute and transient psychotic disorder (F23) according to ICD-10 criteria (International Classification of Diseases; WHO, 1993) were recruited

\footnotetext{
11 is added because the subcategory which is accessed first can never be a switch (because there is no preceding subcategory), but it can be a cluster.
} 
from the University Psychiatric Hospital 'Vrapče' of the University of Zagreb. Descriptive data are available in Table 1. All patients were in the young adult range. Nineteen patients were diagnosed with an acute schizophrenia-like psychotic disorder (F23.2), while three patients were diagnosed with an acute polymorphic psychotic disorder with symptoms of schizophrenia (F23.1). All patients experienced only one psychotic episode in their lifetime, and their first psychotic episode was the reason for their current hospitalization (cf. Folnegović et al., 1994). Thirteen patients were assessed within one month of the first psychotic episode, while others were tested after either one $(\mathrm{N}=3)$, two $(\mathrm{N}=2)$, three $(\mathrm{N}=2)$, four $(\mathrm{N}=1)$, or five months $(\mathrm{N}=1)$.

\section{[Table 1 near here]}

All patients were receiving antipsychotics. The daily dosages of different antipsychotics were transformed into chlorpromazine equivalents (CPZE) (Danivas \& Venkatasubramanian, 2020). Nine patients were receiving intermediate (400-799 mg), while 13 patients were receiving low daily antipsychotic dosages (0-399 mg; Dudley et al., 2017). Fourteen patients were receiving two antipsychotics. Of these, eight were receiving a combination of a typical and an atypical antipsychotic, while six were receiving two atypical antipsychotics. The eight patients receiving monoantipsychotic therapy were all receiving atypical antipsychotics. Sixteen patients were receiving concomitant psychopharmacological treatment. All patients receiving concomitant treatment were receiving benzodiazepines. Six patients were receiving anticholinergics. Two patients each were receiving mood stabilizers, antidepressants, and hypnotics.

22 Croatian-speaking healthy subjects (HS) were recruited for the comparison group. HS were matched with PwFEP in age, the distribution of sexes, and handedness. All HS denied having histories of psychiatric and neurological disorders. Ethical approval for the study was obtained by 
the Ethical Committee of the University Psychiatric Hospital 'Vrapče' (no. 23-305/8-18). All subjects signed an informed consent form.

\subsection{Semantic fluency}

Semantic fluency was administered using five semantic categories as cues: animals, trees, vegetables, fruits, and musical instruments. Subjects were told to name as many members of the given category as they could. Cues were given aurally and verbally by an experimenter. The time limit was set at 60 seconds. The subjects' outputs were audio-recorded and subsequently transcribed for analyses.

The following dependent variables were used: (1) the raw number of correct words, (2) perseveration rate, (3) intrusion rate, (4) switching rate, and (5) cluster magnitude. We calculated both the total scores across the five subtasks and individual scores for the five subtasks. Incorrect words included errors (perseverations and intrusions) and superordinate words. Superordinate words were, by their definition, only classified as such if the subject named both the superordinate words (e.g. bird) and a bird member (e.g. ostrich), but not if they produced, e.g. the word bird, but no instances of the concept BIRD. Sex variations of animal names (e.g. cow-bull) were classified as correct. Perseverations included repetitions of words (regardless of their position), while intrusions encompassed words that denoted non-members of the given category (e.g. apple in the vegetable subtask). The perseveration and intrusion rates were calculated by dividing the raw number of perseverations and intrusions, respectively, by the total output.

Clusters were defined as groups of at least two successive words belonging to the same semantic subcategory of the given category. Switches included words that denoted a concept belonging to a 
different semantic subcategory of the given category compared to the preceding word (Troyer et al., 1997). The switching rate was calculated by dividing the raw number of non-clustered switches by the raw number of times a new subcategory was accessed (i.e. raw number of switches plus one for the first subcategory, which can never be a switch). Cluster magnitude was calculated by dividing the raw number of clustered words by the raw number of clusters. Subcategories for the category animals included African animals, farm animals, pets, birds, reptiles, insects, fish, exotic animals, etc. (Troyer 2000: 377-378), for the category vegetables root/tubers, fruit-like vegetables, green stem, spices, flower vegetables, peas/beans/grains, etc. (Rosselli et al. 2009: 727), for the category fruits sweet fruits, acid fruits, nuts, citrus fruits, tropical fruits, forest fruits, nuts, etc. (Jaimes-Bautista et al. 2020: 5; Rosselli et al. 2009: 726), and for the category musical instruments string instruments, brass instruments, percussion instruments, wind instruments, etc. (Hirshorn \& Thompson-Schill 2006: 2549). We were not aware of any conventions for analyzing clusters for the category trees so these subcategories were determined a posteriori following the techniques of previous studies: evergreens, deciduous trees, decorative trees, exotic trees, fruit trees, etc. The same tree subcategories were used in Gabrić \& Vandek (2021). Three raters independently analyzed the data. All disagreements and uncertainties were resolved via discussion between the raters.

\subsection{Statistics}

Statistical analyses were conducted in JASP (JASP Team, 2020). Because a considerable part of the data across the five SF subtasks was non-normal in distribution and highly skewed, nonparametric tests were applied. The total scores across the five SF subtasks were compared between 
groups using the Mann-Whitney $U$ test with the significance level set at .05 . Subsequently, the Kruskal-Wallis test was conducted with the independent variable group (HS vs. PwFEP). Post hoc comparisons were conducted using the Mann-Whitney U test. A Bonferroni correction was applied by dividing the alpha value by the number of group comparisons for each dependent variable, with the significance level thus set at $0.01(0.05 / 5)$. The effect size for the Mann-Whitney U test is given by the matched rank biserial correlation. Because no HS produced intrusions on the animal subtask, the post hoc comparison between groups for this variable was conducted using the onesample Wilcoxon signed-rank test with the test value set at $0 \pm 1$. Associations between age, the clinical variables, and the SF variables were analyzed using Spearman correlation coefficients.

\section{Results}

\subsection{Sociodemographic data}

There were no significant correlations between age and the two clinical variables (both $\mathrm{p}>0.100$ ). There were no significant correlations between age and the SF total scores and no significant differences between sexes in the total SF scores neither in the two groups combined nor in the individual groups (all $\mathrm{p}>0.100)$.

\subsection{Clinical data}

Illness duration and daily antipsychotic dosage were significantly negatively correlated $(\rho=-$ $0.564, \mathrm{p}=0.006)$. There were no significant correlations between the two clinical variables and 
the total SF scores (all $\mathrm{p}>0.100$ ). There were no significant differences in the total SF scores between patients tested within the first month after the episode and those tested in the period afterwards, between patients differing in the level of the daily antipsychotic dosage, the number of antipsychotics, and type of antipsychotics, as well as between patients receiving and not receiving concomitant psychopharmacological therapy (all $\mathrm{p}>0.100$ ).

\subsection{Semantic fluency}

\section{[Table 2 near here]}

Comparisons of the total SF scores across the five categories between PwFEP and HS are shown in Table 2. All between-group differences were significant. PwFEP produced both perseverations and intrusions at significantly higher rates, indicating poorer performance monitoring and looseness of the semantic categories' boundaries, respectively. PwFEP further switched at significantly higher rates and built significantly smaller clusters than HS, indicating dysfunctional connectivity between co-hyponymic concepts in the given semantic categories. The effect size was larger for cluster magnitude compared to switching rate, possibly indicating that clustering ability at higher rates (> 2 words) might be particularly impaired within the clustering deficit (i.e. switching surplus) as a whole in PwFEP.

\section{[Table 3 near here]}

Results of the Kruskal-Wallis test testing the effect of group on SF performance are shown in Table 3. The effect of group was significant for all SF variables. Post hoc comparisons revealed that PwFEP produced significantly less correct words on all subtasks (animals: $U=65.5, \mathrm{p}<0.001, \mathrm{r}_{\mathrm{b}}$ 
$=0.729$; musical instruments: $\mathrm{U}=72.0, \mathrm{p}<0.001, \mathrm{r}_{\mathrm{b}}=0.702$; fruits: $\mathrm{U}=73.5, \mathrm{p}<0.001, \mathrm{r}_{\mathrm{b}}=$ 0.696; trees: $\mathrm{U}=117.0, \mathrm{p}=0.003, \mathrm{r}_{\mathrm{b}}=0.517$; vegetables: $\mathrm{U}=117.5, \mathrm{p}=0.003, \mathrm{r}_{\mathrm{b}}=0.514$ ), with the effect sizes somewhat higher for the animal, musical instrument, and fruit compared to the tree and vegetable subtasks.

Although PwFEP and HS differed in the total perseveration and intrusion rates in the aggregate score and the effects of group on both variables were significant, there were no significant differences between the two groups in these variables in the post hoc comparisons after the Bonferroni correction. The highest effect sizes were found for the perseveration rate on the animal subtask $\left(\mathrm{U}=316.5, \mathrm{p}=0.027, \mathrm{r}_{\mathrm{b}}=0.308\right)$ and the intrusion rate on the tree subtask $(\mathrm{U}=312.5, \mathrm{p}$ $\left.=0.042, \mathrm{r}_{\mathrm{b}}=0.291\right)$.

PwFEP displayed a significantly higher total switching rate, yet none of the post hoc comparisons reached significance after Bonferroni correction. The comparison of performances in the vegetable subtask turned insignificant after the Bonferroni correction $\left(\mathrm{U}=328.0, \mathrm{p}=0.045, \mathrm{r}_{\mathrm{b}}=0.355\right)$. Descriptive data showed that PwFEP tended to switch at higher rates compared to HS on all subtasks. However, the mean difference between groups for the tree $(0.7 \%)$ subtask was trivial, as well as lower compared to the mean differences for the vegetable (12.7), musical instrument (8.7), animal (8.4), and fruit subtasks (4.7).

PwFEP had significantly smaller cluster magnitudes compared to HS in the animal ( $U=94.5, p<$ $\left.0.001, \mathrm{r}_{\mathrm{b}}=0.610\right)$, musical instrument $\left(\mathrm{U}=94.5, \mathrm{p}<0.001, \mathrm{r}_{\mathrm{b}}=0.610\right)$, and vegetable subtasks $(\mathrm{U}$ $\left.=106.5, \mathrm{p}<0.001, \mathrm{r}_{\mathrm{b}}=0.560\right)$. The comparison of performances in the fruit subtask turned insignificant after the Bonferroni correction $\left(\mathrm{U}=153.5, \mathrm{p}=0.036, \mathrm{r}_{\mathrm{b}}=0.366\right)$. Additionally, similarly to the analyses of the total SF scores, the effect of group was stronger for cluster 
magnitude compared to the switching rate, again possibly indicating that clustering at higher rates might be more impaired than the clustering ability as a whole. Data for cluster magnitude are visually summarized in Figures 1.

[Figure 1 near here]

\section{Discussion}

As anticipated, PwFEP produced significantly less correct words both in the aggregate score and across the five subtasks and on the individual subtasks, as shown by the post hoc comparisons. These and other results appeared unaffected by sociodemographic factors as (1) PwFEP and HS were matched in age, sex, self-reported handedness, and education and (2) inferential statistical analyses revealed no effects of age or sex on SF performance in our study. This result is in line with the current evidence suggesting that both chronic and first-episode patients display cognitive deficits (Mesholam-Gately et al., 2009) and with previous studies reporting deficits in productivity on SF (Blessing et al., 2009; Chou et al., 2015). Furthermore, PwFEP had significantly higher perseveration rates, indicating a diminished capacity for performance monitoring and possible impairments in working memory, as well as significantly higher intrusion rates, indicating that the boundaries of semantic categories are looser compared to HS. The latter result agrees with studies demonstrating abnormally broad semantic spreading activation on semantic tasks in patients with schizophrenia (Assaf et al., 2006; Spitzer, 1997). Nevertheless, none of the post hoc comparisons for the two error variables was significant after the Bonferroni correction. 
Regarding switching and clustering, PwFEP had a significantly higher switching rate compared to HS in the aggregate score across the five subtasks. Still, no significant differences were found in the post hoc comparisons. Moreover, PwFEP produced significantly smaller clusters in the aggregate score. The post hoc comparisons between-group differences were significant for the animal, musical instrument, and vegetable, but not the tree and fruit subtasks. Although none of the post hoc comparisons in the switching rate achieved significance, the mean difference between groups on the tree task was trivial (0.7) and smaller compared to other subtasks, suggesting that both the switching rate and cluster magnitude on the tree subtask might be relatively normal in PwFEP. Importantly, in both the analyses of the aggregate scores and the Kruskal-Wallis test, the effect of group was greater for cluster magnitude than for the switching rate, suggesting greater impairments in cluster magnitude compared to the relative cluster frequency.

The smaller cluster magnitude indicates that PwFEP displayed less efficient connectivity between co-hyponymic concepts in an accessed subcategory of the given subtask category (e.g. cohyponyms elephant-lion-crocodile of the subcategory African animals). Consequences of this presumed less efficient connectivity are (1) higher risk of not building a cluster once a switch has been generated and (2) higher risk of switching after two clustered words have been uttered. It might be further suggested that the higher switching rate in PwFEP indicates relatively intact connectivity between co-hyponymic concepts on the category level (e.g. co-hyponyms birdsreptiles-farm animals of the category animals). However, a higher intrusion rate and a deficit in the raw total number of switches $\left(\mathrm{U}=144.5, \mathrm{p}=0.023, \mathrm{r}_{\mathrm{b}}=0.403\right.$, not reported in the Results) indicate that the connectivity between co-hyponymic concepts on the category level is also relatively dysfunctional in PwFEP, albeit less pronounced compared to the level of subcategorical co-hyponyms. Further, although PwFEP also tended to produce fewer non-clustered switches (total 
score) compared to HS (mean difference: 3.954), there were no significant differences between groups in the total raw number of non-clustered switches $\left(\mathrm{U}=144.5, \mathrm{p}=0.121, \mathrm{r}_{\mathrm{b}}=0.275\right)$. However, when we excluded the errors from the raw number of non-clustered switches, the difference between groups turned significant (mean difference: 6.454; $\mathrm{U}=128.0, \mathrm{p}=0.008, \mathrm{r}_{\mathrm{b}}=$ 0.471), indicating that although switching may be more spared compared to cluster magnitude, there are clearly dysfunctions in the connectivity between co-hyponymic subcategories of a given semantic category as well.

Our results further show that there are possibly category-specific effects on the deficits in cluster magnitude in PwFEP. As reported in the Introduction, significantly smaller clusters in patients with schizophrenia during 60-second SF have been found on animal fluency tasks (Berberian et al., 2016), but not in a study analyzing the aggregate scores of the categories animals, fruits, and objects (Bozikas et al., 2005). Importantly, we found no significant differences in cluster magnitude on the tree and fruit subtasks, confirming the possibility that patients with schizophrenia (and FEP for that matter) might not show significantly clustering deficits when the category fruits is used. Notably, the mean difference between groups in the switching rate on the tree subtask was only $0.7 \%$, supporting the hypothesis of the tree subtask's specificity. Since studies have suggested that performance on the tree but not the animal subtask is associated with executive functions in healthy subjects (Gabrić \& Vandek, 2020; Vandek et al., 2018) and since PwFEP in our sample demonstrated no significant differences in cluster magnitude on the tree subtask, it is possible that patients with FEP (and, by extension, schizophrenia) display less pronounced or no clustering deficits on semantic fluency tasks which engage executive functioning rather than relatively automatic semantic processing. However, other hypotheses are possible as well. Firstly, the lack of significant differences in the clustering magnitude on the tree and fruit subtasks might 
reflect the fact that subjects tend to cluster less on these categories compared to other categories (such as animals). Indeed, HS produced fewer words per cluster on the tree and fruit compared to animal and musical instrument subtasks. However, if we were to hypothesize that PwFEP might display statistically normal clustering on categories on which subjects typically cluster less, we would expect no clustering deficits on the vegetable subtask because both HS and PwFEP produced the smallest clusters on this subtask. Contrary to this expectation, the clustering deficits on the vegetable subtask were statistically significant and comparable to the deficits on the animal and musical instrument subtasks. Furthermore, one previous study reported disorganized semantic clustering in patients with Parkinson's disease on the fruit but not animal subtask (Tagini et al., 2018), indicating that deficits in semantic clustering may be observable even in semantic categories on which subjects presumably tend to produce smaller clusters. Secondly, clustering performances on the tree subtask may presumably vary more between different speakers (healthy or not) because our ability to form networks of subcategories and subcategorical co-hyponyms in our semantic memory rests on our experiences. Arguably, people who spend significant portions of their lifetimes in rural compared to urban areas might produce larger tree clusters. Similarly, older people in Croatia may also produce larger tree clusters compared to younger people given the increasing rate of urbanization. These aspects might have modulated clustering performances in our study, especially given that all of the subjects in our study were young adults and that all healthy subjects were situated in Zagreb (mostly urban area) at the time of the assessments.

On the other hand, the lack of statistically significant differences on the tree and fruit subtasks might simply have been spurious. While this may be true, it is nevertheless apparent from the descriptive and inferential statistical data that there is some gradation in the extent of the clustering deficits across the five semantic categories. Specifically, the mean difference in cluster magnitudes 
on the tree subtask was 0.278 (amounting to $10.3 \%$ ), while on the vegetable task it was 0.445 (18.4\%, without controlling for the fact that clusters were smaller on the vegetable compared to the tree subtask). What is more, the mean differences on the animal and musical instrument subtasks were 0.784 (21.8\%) and 1.098 (31.4\%), respectively. Thus, although PwFEP did in fact produce fewer words per cluster on the tree subtask, this statistically non-significant deficit was much less pronounced compared to some other categories from the perspective of descriptive data and effect sizes.

It is interesting that the naturalness of the category does not appear to have been in association with the extent of the clustering deficits in PwFEP. Specifically, the extent of the clustering deficit was very similar for the animal and musical instrument subtasks (effect sizes $-r_{b} s-$ were 0.610 for both comparisons), tentatively suggesting that PwFEP might display comparable clustering deficits on both natural and man-made semantic (or animate and inanimate) categories (cf. Sekulić Sović et al., 2019). Nevertheless, we tested only one man-made semantic category and future research on this issue is thus needed.

Regarding the hypothesis of abnormally broad semantic spreading activation in schizophrenia, although our results are in agreement with the hypothesis, they can be interpreted by other phenomena as well, such as selective degradation of the semantic memory. Be that as it may, if we assume that the deficits observed in our study were due to abnormally broad semantic spreading activation, our results suggest that (1) the broader co-activation is not drastically broad, i.e. it relatively infrequently (but still significantly more often than in HS) surpasses the category's boundaries (indicated by the rates of intrusions; cf. Erdeljac et al., 2019) and that (2) its degree on SF tasks is category-dependent. Furthermore, because most studies with chronic patients with schizophrenia failed to find significant differences in clustering and switching strategies compared 
to healthy subjects (Bozikas et al., 2005; Okruszek et al., 2013), it is possible that this kind of phenomenon in the context of SF performance is not necessarily associated with the diagnosis of schizophrenia or the presence of positive symptoms but perhaps with other clinical factors such as a recent history of a psychotic episode or recent illness onset (< one month). A previous study on SF in schizophrenia found that the SF output is disorganized in patients with an earlier age of illness onset (<45; Paulsen et al., 1996). Unfortunately, we collected no follow-up data to identify patients who subsequently met ICD-10 criteria for schizophrenia so it remains unclear whether the eventual abnormally broad semantic spreading activation might be associated with an earlier onset of schizophrenia.

\section{Limitations}

Firstly, the sample size was small. Secondly, we did not investigate the possible influence of psychotic and other symptoms on performance. Thirdly, we focused on natural semantic categories, failing to systematically investigate eventual differences in the processing of semantic categories differing in the level of naturalness, animacy, concreteness, etc. Fourthly, we did not test the subjects on other neuropsychological assessments apart from SF, thus leaving open the question of whether the observed SF deficits are associated with other cognitive dysfunctions.

\section{Conclusion}

Patients with first-episode psychosis (PwFEP) produced significantly fewer correct words in the aggregate score and across all categories. The switching rate was significantly higher in PwFEP, 
but no post hoc comparisons were significant. PwFEP also produced significantly smaller clusters, yet the post hoc comparisons for the tree and fruit task were not significant. A higher switching rate and smaller clusters indicate less efficient functional connectivity within a subcategory of the given category, but not necessarily between subcategories of the given category. Although both less likely to produce a cluster once a switch has been uttered and to produce clusters larger than two words compared to healthy subjects, the latter deficit was more pronounced. Finally, our results also suggest that there might be category-specific effects on the clustering deficit in PwFEP, but future research is needed to clarify this.

\section{Acknowledgements}

We thank Ninoslav Mimica and Aleksandar Savić from the University Psychiatric Hospital 'Vrapče' of the University of Zagreb for making this research possible, allowing us to work with patients at the Hospital, as well as their generous insight. We further thank them for their assistance in data collection, data analyses, and interpretations relating to psychiatric practice. We also thank Vlasta Erdeljac and Martina Sekulić Sović from the Department of Linguistics of the University of Zagreb for inviting us and encouraging us to work on these data.

\section{Funding}

This work was supported by the Faculty of Humanities and Social Sciences of the University of Zagreb under Grant 11-831-1006. 


\section{Declaration of interest statement}

The authors report no conflict of interest.

\section{References}

1. Allen, H. A., Liddle, P. F., \& Frith, C. D. (1993). Negative features, retrieval processes and verbal fluency in schizophrenia. British Journal of Psychiatry, 163(6), 769-775. https://doi.org/10.1192/bjp.163.6.769

2. Aloia, M. S., Gourovitch, M. L., Weinberger, D. R., \& Goldberg, T. E. (1996). An investigation of semantic space in patients with schizophrenia. Journal of the International Neuropsychological Society, 2(4), 267-273. https://doi.org/10.1017/S1355617700001272

3. Amunts, J., Camilleri, J. A., Eickhoff, S. B., Heim, S., \& Weis, S. (2020). Executive functions predict verbal fluency scores in healthy participants. Scientific Reports, 10, 11141. https://doi.org/10.1038/s41598-020-65525-9

4. Batty, R., Francis, A., Thomas, N., Hopwood, M., Ponsford, J., Johnston, L., \& Rossell, S. (2015). Verbal fluency, clustering, and switching in patients with psychosis following traumatic brain injury (PFTBI). Psychiatry Research, 227(2-3), 152-159. https://doi.org/10.1016/j.psychres.2015.03.040

5. Berberian, A. A., Moraes, G. V., Gadelha, A., Brietzke, E., Fonseca, A. O., Scarpato, B. S., Vicente, M. O., Seabra, A. G., Bressan, R. A., \& Lacerda, A. L. (2016). Is semantic verbal fluency impairment explained by executive function deficits in schizophrenia?. Jornal Brasileiro de Psiquiatria, 38, 121-126. https://doi.org/10.1590/1516-4446-2015$\underline{1663}$ 
6. Berto, G., \& Galaverna, F. S. (2016). Semantic memory organization on verbal fluency test "Human Body Parts" in patients with chronic schizophrenia diagnosis and healthy controls. European Journal of Psychiatry, 30(2), 97-108. http://scielo.isciii.es/scielo.php?script=sci_arttext\&pid=S0213-61632016000200001

7. Biesbroek, J. M., van Zandvoort, M. J. E., Kapelle, L. J., Velthius, B. K., Biessels, G. J., \& Postma, A. (2016). Shared and distinct anatomical correlates of semantic and phonemic fluency revealed by lesion-symptom mapping in patients with ischemic stroke. Brain Structure and Function, 221, 2123-34. https://doi.org/10.1007/s00429-015-1033-8

8. Blessing, A., Wangelin, B., Regehr, H., \& Rudolph, N. (2009). Verbal fluency tasks in first-episode psychosis. Clinical Neuropsychiatry, 6(1), 21-28. https://psycnet.apa.org/record/2009-06871-003

9. Bozikas, V. P., Kosmidis, M. H., \& Karavatos, A. (2005). Disproportionate impairment in semantic verbal fluency in schizophrenia: differential deficit in clustering. Schizophrenia Research, 74, 51-59. https://doi.org/10.1016/j.schres.2004.05.001

10. Breitborde, N. J. K., Srihari, V. H., \& Woods, S. W. (2009). Review of the operational definition for first-episode psychosis. Early Intervention in Psychiatry, 3(4), 259-265. https://doi.org/10.1111/j.1751-7893.2009.00148.x

11. Chen, R. Y. L., Chen, E. Y. H., Chan, C. K. Y., Lam, L. C. W., \& Lieh-Mak, F. (2000). Verbal fluency in schizophrenia: reduction in semantic store. Australian and New Zealand Journal of Psychiatry, 34, 43-48. https://doi.org/10.1046/j.1440-1614.2000.00647.x

12. Danivas, V., \& Venkatasubramanian, G. (2020). Current perspectives on chlorpromazine equivalents: comparing apples and oranges!. Indian Journal of Psychiatry, 55(2), 207-208. https://doi.org/10.4103/0019-5545.111475 
13. Dudley, K., Liu, X., \& De Haan, S. (2017). Chlorpromazine dose for people with schizophrenia. Cochrane Database of Systematic Reviews, 4(4), CD007778. https://doi.org/10.1002/14651858.CD007778.pub2

14. Elvevåg, B., Fisher, J. E., Gurd, J. M., \& Goldberg, T. E. (2002). Semantic clustering in verbal fluency: schizophrenic patients versus control participants. Psychological Medicine, 32(5), 909-917. https://doi.org/10.1017/S0033291702005597

15. Erdeljac, V., Kužina, I., Vandek, M., Sekulić Sović, M., Mimica, N., Ostojić, D., \& Savić, A. (2019). Semantic and pragmatic relations in categorization in early course psychosis. In: V. Erdeljac \& M. Sekulić Sović (eds.), Interdisciplinary Linguistics and Psychiatric Research on Language Disorders (pp. 55-67). FF Press. https://doi.org/10.17234/9789531758314.05

16. Folnegović, Z., Hrabak-Zerjavić, V., Šmalc-Folnegović, \& Mimica, N. (1994). Hospital incidence of schizophrenia in Croatia over a 25-year period. Schizophrenia Research, 11(2), 99-100. https://www.sciencedirect.com/science/article/pii/0920996494902488

17. Fong, M. C. M., Hui, N. Y., Fung, E. S. W., Ma, M. K. H., Law, T. S. T., Wang, X., \& Wang, S. W. (2020). Which cognitive functions subserve clustering and switching in category fluency? Generalisations from an extended set of semantic categories using linear mixed-effects modelling. Quarterly Journal of Experimental Psychology, 73(12), 21322147. https://doi.org/10.1177/1747021820957135

18. Gabrić, P. (2021a). Comment on "Semantic memory impairment across the schizophrenia continuum: a meta-analysis of category fluency performance" by Tan et al. [Preprint]. PsyArXiv. https://doi.org/10.31234/osf.io/jenpt 
19. Gabrić, P. (2021b). Continued methodological imprecisions, nontransparency, and misinterpretation in a meta-analysis of semantic fluency in schizophrenia: a comment on "Corrigendum to: Semantic memory impairment across the schizophrenia continuum: a meta-analysis of category fluency performance" by Tan et al. [Preprint]. PsyArXiv. https://doi.org/10.31234/osf.io/ayfb7

20. Gabrić, P., \& Vandek, M. (2020, October 21-24). Quantitative and qualitative differences in performance within the semantic and letter fluency tasks [Poster presentation]. 12th Annual Meeting of the Society for the Neurobiology of Language, Online, US. https://doi.org/10.5281/zenodo.4681799

21. Gabrić, P., \& Vandek, M. (2021). Striking differences in performance patterns and neuropsychological correlates within semantic and phonemic fluency tasks [Preprint]. PsyArXiv. https://doi.org/10.31234/osf.io/jykpu

22. Giovannetti, T., Goldstein, R. Z., Schullery, M., Barr, W. B., Bilder, R. M. (2003). Category fluency in first-episode schizophrenia. Journal of the International Neuropsychological Society, 9, 384-393. https://doi.org/10.10170S1355617703930049

23. Gourovitch, M. L., Goldberg, T. E., \& Weinberger, D. R. (1996). Verbal fluency deficits in patients with schizophrenia: semantic fluency is differentially impaired as compared with phonologic fluency. Neuropsychology, 10(4), 573-577. https://doi.org/10.1037/0894$\underline{4105.10 .4 .573}$

24. Hirshorn, E. A., \& Thompson-Schill, S. L. (2006). Role of the left inferior frontal gyrus in covert word retrieval: neural correlates of switching during verbal fluency. Neuropsychologia, $\quad 44(12), \quad 2547-2557$. https://doi.org/10.1016/j.neuropsychologia.2006.03.035 
25. Jaimes-Bautista, A. G., Rodríguez-Camacho, M., Martínez-Juárez, I. E., \& RodríguezAgudelo, Y. (2020). Quantitative and qualitative analysis of semantic verbal fluency in patients with temporal lobe epilepsy. Neurología (English Edition), 35(1), 1-9. https://doi.org/10.1016/j.nrleng.2019.03.006

26. JASP Team (2020). JASP (Version 0.13.1) [Computer software]. https://jasp-stats.org/

27. Joyce, E. M., Collinson, S. L., \& Crichton, P. (1996). Verbal fluency in schizophrenia: relationship with executive function, semantic memory and clinical alogia. Psychological Medicine, 26, 39-49. https://doi.org/10.1017/S0033291700033705

28. Kosmidis, M. H., Bozikas, V. P., Vlahou, C. H., Kiosseoglou, G., Giaglis, G., \& Karavatos, A. (2005). Verbal fluency in institutionalized patients with schizophrenia: Age-related performance decline. Psychiatry Research, 134(3), 233-240. https://doi.org/10.1016/j.psychres.2005.02.003

29. Kuperberg, G. R., Delaney-Busch, N., Fanucci, K., \& Blackford, T. (2018). Priming production: neural evidence for enhanced automatic semantic activity preceding language production in schizophrenia. NeuroImage: Clinical, 18, 74-85. https://doi.org/10.1016/j.nicl.2017.12.026

30. Landrø, N. I., \& Ueland, T. (2008). Verbal memory and verbal fluency in adolescents with schizophrenia spectrum disorders. Psychiatry and Clinical Neurosciences, 62(6), 653-661. https://doi.org/10.1111/j.1440-1819.2008.01864.x

31. Lemieux, P., \& Macoir, J. (2017). Fonctions cognitives sous-jacentes aux déficits de fluence verbale dans la schizophrénie: revue de la littérature. Annales Médicopsychologiques, $\quad$ revue psychiatrique, 175(2), 127-139. https://doi.org/10.1016/j.amp.2016.06.002 
32. Menezes, N. M., Arenovich, T., \& Zipursky, R. B. (2006). A systematic review of longitudinal outcome studies of first-episode psychosis. Psychological Medicine, 36(10), 1349-1362. https://doi.org/10.1017/s0033291706007951

33. Mesholam-Gately, R. I., Giuliano, A. J., Goff, K. P., Faraone, S. V., \& Seidman, L. J. (2009). Neurocognition in first-episode schizophrenia: a meta-analytic review. Neuropsychology, 23(3), 315-336. https://doi.org/10.1037/a0014708

34. Moore, D. J., Savla, G. N., Woods, S. P., Jeste, D. V., \& Palmer, B. W. (2006). Verbal fluency impairments among middle-aged and older outpatients with schizophrenia are characterized by deficient switching. Schizophrenia Research, 87(1-3), 254-260. https://doi.org/10.1016/j.schres.2006.06.005

35. Moreno-Martínez, F. J., Ruiz, M., \& Montoro, P. R. (2017). Why almost always animals? Ranking fluency tasks for the detection of dementia based on receiver operating characteristic (ROC) and quality ROC analyses. Dementia and Geriatric Cognitive Disorders, 43(1-2), 59-70. https://doi.org/10.1159/000454916

36. Nagels, A., Kircher, T., Dietsche, B., Backes, H., Marquetand, J., \& Krug, A. (2012). Neural processing of overt word generation in healthy individuals: the effect of age and $\begin{array}{llll}\text { word } & \text { knowledge. } & \text { NeuroImage, } & 61,\end{array}$ https://doi.org/10.1016/j.neuroimage.2012.04.019

37. Neill, E., Gurvich, C., \& Rossell, S. L. (2014). Category fluency in schizophrenia research: is it an executive or semantic measure?. Cognitive Neuropsychiatry, 19(1), 81-95. https://doi.org/10.1080/13546805.2013.807233

38. Neves, T. R. F., Araújo, N. B. de, Silva, F. de O., Ferreira, J. V. A., Nielsen, T. R., Engedal, K., Laks, J., \& Deslandes, A. C. (2020). Accuracy of the semantic fluency test to separate 
healthy old people from patients with Alzheimer's disease in a low education population. Jornal Brasileiro de Psiquiatria, 69(2), 82-87. https://doi.org/10.1590/0047$\underline{2085000000270}$

39. Nuyen, J., Sitskoorn, M. M., Cahn, W., \& Kahn, R. S. (2005). Verbal memory in firstepisode schizophrenia: heterogeneity in performance?. Journal of the International Neuropsychological Society, 11, 152-162. https://doi.org/10.1017/s1355617705050162

40. Okruszek, Ł., Rutkowska, A., \& Wilińska, J. (2013). Clustering and switching strategies during the semantic fluency task in men with frontal lobe lesions and in men with schizophrenia. Psychology of Language and Communication, 17(1), 93-100. https://doi.org/10.2478/plc-2013-0006

41. Paulsen, J. S., Romero, R., Chan, A., Davis, A. V., Heaton, R. K., \& Jeste, D. V. (1996). Impairment of the semantic network in schizophrenia. Psychiatry Research, 63(2-3), 109121. https://doi.org/10.1016/0165-1781(96)02901-0

42. Pekkala, S. (2012). Verbal fluency tasks and the neuropsychology of language. In: M. Faust (ed.), The Handbook of the Neuropsychology of Language (pp. 619-634). WileyBlackwell. https://doi.org/10.1002/9781118432501.ch30

43. Phillips, T. J., James, A. C. D., Crow, T. J., \& Collinson, S. L. (2004). Semantic fluency is impaired but phonemic and design fluency are preserved in early-onset schizophrenia. Schizophrenia Research, 70, 215-222. https://doi.org/10.1016/j.schres.2003.10.003

44. Popescu, C. A., \& Micluţia, I. V. (2006). Semantic fluency in schizophrenia. Journal of Evidence-Based Psychotherapies, $\quad 6(2), \quad$ 105-118. http://jebp.psychotherapy.ro/vol6no2/semantic-fluency-in-schizophrenia 
45. Popescu, C. A., Miclutia, I. V., Macrea, R., Craciun, I., \& Zaharia, A. (2007). Semantic fluency in schizophrenia. European Psychiatry, 22, S133. https://doi.org/10.1016/j.eurpsy.2007.01.426

46. Reed, S. I. (2008). First-episode psychosis: a literature review. International Journal of Mental Health Nursing, 17(2), 85-91. https://doi.org/10.1111/j.1447-0349.2008.00515.x

47. Rinaldi, R., Lefebvre, L., \& Trappeniers, J. (2014). Language, executive functioning and symptomatology-Is fluency a transversal tool in schizophrenia?. Open Journal of Psychiatry, 3(4), 358-369. https://doi.org/10.4236/ojpsych.2013.34038

48. Robert, P. H., Lafont, V., Medecin, I., Berthet, L., Thauby, S., Baudu, C., \& Darcourt, G. (1998). Clustering and switching strategies in verbal fluency tasks: comparison between schizophrenics and healthy adults. Journal of the International Neuropsychological Society, 4(6), 539-546. https://doi.org/10.1017/S1355617798466025

49. Rosenkranz, A., Kircher, T., \& Nagels, A. (2019). Neuropsychological correlates underlying verbal fluency deficits in schizophrenia: the role of attention and executive function. In: V. Erdeljac, \& M. Sekulić Sović (eds.), Interdisciplinary Linguistics and Psychiatric Research on Language Disorders (pp. 45-54). FF Press. https://doi.org/10.17234/9789531758314.04

50. Rossell, S. L., Rabe-Hesketh, S., Shapleske, J., \& David, A. S. (1999). Is semantic fluency differentially impaired in schizophrenic patients with delusions?. Journal of Clinical and Experimental Neuropsychology, 21(5), 629-642. https://doi.org/10.1076/jcen.21.5.629.865

51. Rosselli, M., Tappen, R., Williams, C., Salvatierra, J., \& Zoller, Y. (2009). Level of education and category fluency task among Spanish speaking elders: number of words, 
clustering, and switching strategies. Aging, Neuropsychology, and Cognition, 16(6), 721744. https://doi.org/10.1080/13825580902912739

52. Sekulić Sović, M., Erdeljac, V., Kužina, I., Vandek, M., Mimica, N., Ostojić, D., \& Savić, A. (2019). Shared lexical-semantic features and the animacy effect in early-course psychosis. In: V. Erdeljac, \& M. Sekulić Sović (Eds.), Interdisciplinary Linguistic and Psychiatric Research on Language Disorders (pp. 69-81). FF Press. https://doi.org/10.17234/9789531758314.06

53. Spitzer, M. (1997). A cognitive neuroscience view of schizophrenic thought disorder. Schizophrenia Bulletin, 23(1), 29-50. https://doi.org/10.1093/schbul/23.1.29

54. Stielow, A., \& Stenneken, P. (2017). Der Beitrag exekutiver und sprachlicher Teilleistungen in semantischen und formal-lexikalischen Wortflüssigkeitsaufgaben. Sprache $\cdot$ Stimme $\cdot$ Gehör, 41, 197-203. https://doi.org/10.1055/s-0043-117202

55. Sumiyoshi, C., Ertugrul, A., Yagcioglu, A. E. A., \& Sumiyoshi, T. (2009). Semantic memory deficits based on category fluency performance in schizophrenia: similar impairment patterns of semantic organization across Turkish and Japanese patients. Psychiatry Research, 167(1-2), 47-57. https://doi.org/10.1016/j.psychres.2007.12.009

56. Sung, K., Gordon, B., Vannorsdall, T. D., Ledoux, K., Pickett, E. J., Pearlson, G. D., \& Schretlen, D. J. (2012). Semantic clustering of category fluency in schizophrenia examined with singular value decomposition. Journal of the International Neuropsychological Society, 18, 565-575. https://doi.org/10.1017/S1355617712000136

57. Tagini, S., Seyed-Allaei, S., Scarpina, F., Toraldo, A., Mauro, A., Cherubini, P., \& Reverberi, C. (2018). When fruits lose to animals: disorganized search of semantic memory 
in Parkinson's disease. Neuropsychology, Advance online publication. https://doi.org/10.1037/neu0000429

58. Tan, E. J., Neill, E., Tomlinson, K., \& Rossell, S. L. (2020). Semantic memory impairment across the schizophrenia continuum: a meta-analysis of category fluency performance. $\begin{array}{llll}\text { Schizophrenia Bulletin } & \text { Open, } & \text { 1(1), } & \text { sgaa054. }\end{array}$ https://doi.org/10.1093/schizbullopen/sgaa054

59. Tan, E. J., Neill, E., Tomlinson, K., \& Rossell, S. L. (2021). Corrigendum to: Semantic memory impairment across the schizophrenia continuum: a meta-analysis of category fluency performance. Schizophrenia Bulletin Open, 2(1), sgab018. https://doi.org/10.1093/schizbullopen/sgab018

60. Thiele, K., Quinting, J. M., \& Stenneken, P. (2016). New ways to analyze word generation performance in brain injury: a systematic review and meta-analysis of additional performance measures. Journal of Clinical and Experimental Neuropsychology, 38(7), 764-781. https://doi.org/10.1080/13803395.2016.1163327

61. Troyer, A. K. (2000). Normative data for clustering and switching on verbal fluency tasks. Journal of Clinical and Experimental Neuropsychology, 22(3), 370-378. https://doi.org/10.1076/1380-3395(200006)22:3;1-v;ft370

62. Troyer, A. K., \& Moscovitch, M. (2006). Cognitive processes of verbal fluency tasks. In: A. M. Poreh (Ed.), Studies on Neuropsychology, Neurology and Cognition: The Quantified Process Approach to Neuropsychological Assessment (pp. 143-160). Taylor \& Francis. https://psycnet.apa.org/record/2007-16443-009 
63. Troyer, A. K., Moscovitch, M., \& Winocur, G. (1997). Clustering and switching as two components of verbal fluency: evidence from younger and older healthy adults. Neuropsychology, 11(1), 138-146. https://doi.org/10.1037//0894-4105.11.1.138

64. Tyburski, E., Sokołowski, A., Chęć, M., Pełka-Wysiecka, J., \& Samochowiec, A. (2015). Neuropsychological characteristics of verbal and non-verbal fluency in schizophrenia patients. Archives of Psychiatric Nursing, 29, 33-38. https://doi.org/10.1016/j.apnu.2014.09.009

65. van Beilen, M., Pijnenborg, M., van Zomeren, E. H., van den Bosch, R. J., Withaar, F. K., \& Bouma, A. (2004). What is measured by verbal fluency tests in schizophrenia?. Schizophrenia Research, 69, 267-276. https://doi.org/10.1016/j.schres.2003.09.007

66. Vandek, M., Gabrić, P., Kužina, I., Erdeljac, V., \& Sekulić Sović, M. (2018, July 2-4). Verbal fluency and working memory interaction [Poster presentation]. 10th International Workshop on Language Production, Nijmegen, Netherlands. https://doi.org/10.5281/zenodo.4681276

67. Veleva, I. I., Stoimenova, M. J., \& Valkova, M. P. (2019). A comparative study of verbal fluency in patients with paranoid schizophrenia, first grade relatives and healthy controls. Archives of the Balkan Medical Union, 54(2), 325-329. https://doi.org/10.31688/ABMU.2019.54.2.16

68. WHO (World Health Organization) (1993). The ICD-10 Classification of Mental and Behavioural Disorders: Clinical Descriptions and Diagnostic Guidelines. World Health Organization. https://www.who.int/classifications/icd/en/bluebook.pdf 
Table 1. Sociodemographic and clinical data.

\begin{tabular}{|c|c|c|}
\hline & Healthy subjects & Patients \\
\hline Age (years) & $\begin{array}{l}23.000(4.220) \\
\quad \text { R } 17-33\end{array}$ & $\begin{array}{l}23.045(4.226) \\
\quad \text { R } 17-33\end{array}$ \\
\hline Males ratio & $14 / 22$ & $14 / 22$ \\
\hline Education & S 19, H 3 & $\mathrm{~S} 22, \mathrm{H} 0$ \\
\hline Handedness (self-report) & $\mathrm{R} 21, \mathrm{~L} 1$ & $\mathrm{R} 21, \mathrm{~L} 1$ \\
\hline Illness duration (months) & & $\begin{array}{l}1.000(1.512) \\
\quad \text { R } 0-5\end{array}$ \\
\hline Daily antipsychotic dosage (CPZE; mg) & & $\begin{array}{l}422.348(195.474) \\
\quad \text { R } 100-800\end{array}$ \\
\hline $\begin{array}{l}\text { Concomitant psychopharmacological } \\
\text { therapy }\end{array}$ & & $16 / 22$ \\
\hline
\end{tabular}

Note: For age, illness duration, and the daily antipsychotic dosage, mean values are reported, alongside the standard deviations in parentheses and ranges in the second row of the cells. Median values for age, illness duration, and the daily antipsychotic dosage were 22,0 , and 400 , respectively. $\mathrm{S}=$ secondary education, $\mathrm{H}=$ higher education, $\mathrm{R}=$ right-handed, $\mathrm{L}=$ left-handed 
Table 2. Comparisons between groups in the aggregate scores across the five SF categories.

\begin{tabular}{|c|c|c|c|}
\hline & Patients & Healthy subjects & Mann-Whitney U test \\
\hline Correct words (raw) & $\begin{array}{c}61.136(13.410) \\
\text { R 39-88 }\end{array}$ & $\begin{array}{c}87.500(17.468) \\
\text { R 54-136 }\end{array}$ & $\mathrm{U}=48.0, \mathrm{p}<0.001, \mathrm{r}_{\mathrm{b}}=0.802$ \\
\hline Perseveration rate $(\%)$ & $\begin{array}{c}4.5(3.9) \\
\text { R } 0.0-15.1\end{array}$ & $\begin{array}{c}1.7(2.3) \\
\text { R } 0.0-9.8\end{array}$ & $\mathrm{U}=367.0, \mathrm{p}=0.003, \mathrm{r}_{\mathrm{b}}=0.517$ \\
\hline Intrusion rate $(\%)$ & $\begin{array}{c}3.0(3.4) \\
\text { R } 0.0-10.9\end{array}$ & $\begin{array}{c}0.7(0.9) \\
\text { R } 0.0-3.0\end{array}$ & $\mathrm{U}=351.0, \mathrm{p}=0.008, \mathrm{r}_{\mathrm{b}}=0.450$ \\
\hline Switching rate $(\%)$ & $\begin{array}{c}60.3(7.8) \\
\text { R } 40.0-72.7\end{array}$ & $\begin{array}{c}53.1(10.4) \\
\text { R 34.2-68.9 }\end{array}$ & $\mathrm{U}=336.5, \mathrm{p}=0.027, \mathrm{r}_{\mathrm{b}}=0.390$ \\
\hline Cluster magnitude & $\begin{array}{l}2.547(2.500) \\
\text { R } 2.118-3.130\end{array}$ & $\begin{array}{l}3.020(2.920) \\
\text { R } 2.308-4.133\end{array}$ & $\mathrm{U}=81.0, \mathrm{p}<0.001, \mathrm{r}_{\mathrm{b}}=0.665$ \\
\hline
\end{tabular}

Note: Mean values are reported, alongside the standard deviations in parentheses and ranges in the second row of the cells. 
Table 3. Descriptive data for the five SF subtasks and results of the Kruskal-Wallis test testing the effect of group on SF performance.

\begin{tabular}{|c|c|c|c|c|c|c|c|c|c|c|c|c|}
\hline & PwFEP & HS & PwFEP & HS & PwFEP & HS & PwFEP & HS & PwFEP & HS & $\mathrm{H}_{(1)}$ & $\mathrm{P}$ \\
\hline Correct words (raw) & $\begin{array}{l}17.227 \\
(5.218)\end{array}$ & $\begin{array}{l}24.591 \\
(4.626)\end{array}$ & $\begin{array}{c}9.409 \\
(4.261)\end{array}$ & $\begin{array}{l}13.455 \\
(4.091)\end{array}$ & $\begin{array}{l}10.864 \\
(3.932)\end{array}$ & $\begin{array}{c}14.273 \\
(4.014)\end{array}$ & $\begin{array}{l}12.818 \\
(3.142)\end{array}$ & $\begin{array}{l}17.455 \\
(3.320)\end{array}$ & $\begin{array}{l}10.818 \\
(4.019)\end{array}$ & $\begin{array}{l}17.727 \\
(6.663)\end{array}$ & 43.391 & $<0.001$ \\
\hline Intrusion rate $(\%)$ & $\begin{array}{c}1.5 \\
(4.5)\end{array}$ & $\begin{array}{c}0.0 \\
(0.0)\end{array}$ & $\begin{array}{c}4.5 \\
(9.8)\end{array}$ & $\begin{array}{c}1.5 \\
(3.0)\end{array}$ & $\begin{array}{c}7.3 \\
(11.4)\end{array}$ & $\begin{array}{c}1.2 \\
(2.8)\end{array}$ & $\begin{array}{c}1.4 \\
(3.1)\end{array}$ & $\begin{array}{c}1.2 \\
(2.6)\end{array}$ & $\begin{array}{c}1.4 \\
(4.1)\end{array}$ & $\begin{array}{c}0.5 \\
(1.6)\end{array}$ & 3.931 & 0.047 \\
\hline Switching rate $(\%)$ & $\begin{array}{c}44.2 \\
(13.9)\end{array}$ & $\begin{array}{c}35.8 \\
(19.5)\end{array}$ & $\begin{array}{c}41.9 \\
(19.9)\end{array}$ & $\begin{array}{c}41.2 \\
(22.9)\end{array}$ & $\begin{array}{c}65.9 \\
(16.4)\end{array}$ & $\begin{array}{c}53.2 \\
(22.6)\end{array}$ & $\begin{array}{c}55.8 \\
(13.7)\end{array}$ & $\begin{array}{c}54.1 \\
(16.3)\end{array}$ & $\begin{array}{c}54.2 \\
(21.9)\end{array}$ & $\begin{array}{c}45.5 \\
(17.6)\end{array}$ & 5.027 & 0.025 \\
\hline
\end{tabular}

Note: Results of the post hoc comparisons are reported in the main text. Mean values are reported. Standard deviations appear in parentheses. 


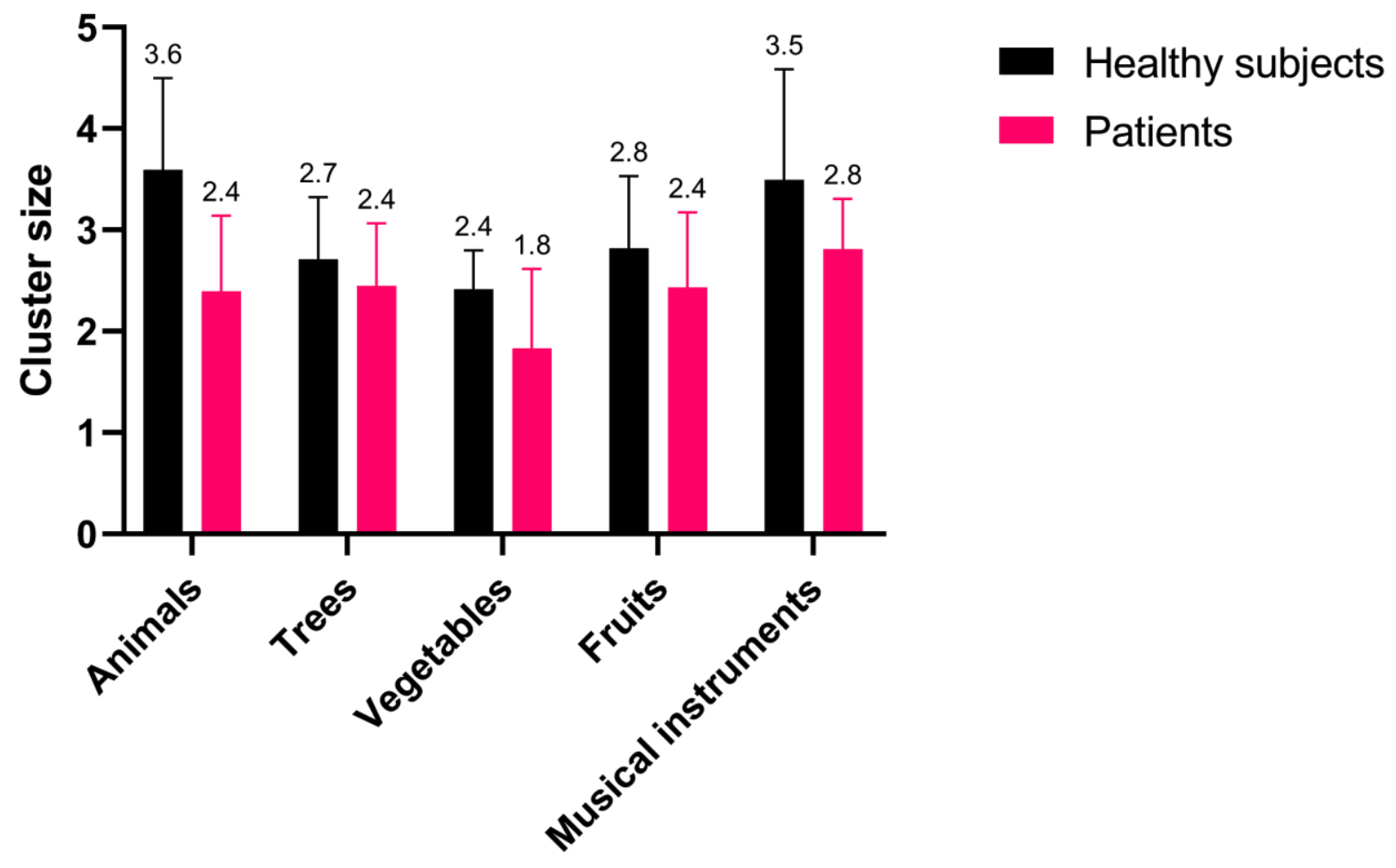

Figure 1. 
Figure 1. A visual summary of the cluster magnitude data in the two groups and across the five SF subtasks. 